\title{
TARMED - neuer Wein in alte Schläuche?
}

\author{
T. Schweizer
}

Es ist erstaunlich. In der schweizerischen Öffentlichkeit gibt es praktisch keine Diskussion und keine Gesamtwürdigung des neuen Ärztetarifs. Dabei ist dieser geradezu ein Jahrhundertwerk. Die je verschiedenen kantonalen Tarifsysteme, welche seit Beginn der sozialen Tarifgebung in der ersten Hälfte des letzten Jahrhunderts im wechselnden Klima des Zeitgeistes aufgewuchert sind, haben den Wert medizinischer Leistungen nur noch sehr verzerrt abgebildet. Man könnte es vielleicht mit der Juragewässerkorrektur vergleichen, aus diesem Sumpf ein einheitliches Tarifsystem gemacht $\mathrm{zu}$ haben, dem eine kohärente und weit herum akzeptierte Philosophie zugrunde liegt. Aber im Gegensatz zu den Kulturingenieuren, wie man die Väter unserer verbesserten Landwirtschaft nannte, kann einer der wichtigsten Begründer des TARMED, H. H. Brunner, nicht damit rechnen, als Standbild auf einem städtischen Platz zu erscheinen. Im Gegenteil: Er wurde fast aufgerieben in einem Zweifrontenkrieg zwischen einerseits einer «Niet-Troika» aus Bundesrätin, santésuisse und Preisüberwacher und andererseits einigen Fachgesellschaften, die ihre Interessen zu spät und letztlich an der falschen Adresse vertraten. Sie hätten sich vor über zehn Jahren mächtiger an den Verhandlungstisch drängen sollen. Wie auch immer: die Probleme sind lösbar. Es war ohnehin klar, dass TARMED nicht von Anfang an «passt», sondern in der Praxis wird erprobt werden müssen. $\mathrm{Ob}$ all der Unzulänglichkeiten und der beträchtlichen politischen Alltagssorgen droht uns Grundsätzliches aus dem Gesichtsfeld zu verschwinden:

Wir haben es bisher versäumt, der Öffentlichkeit die Bedeutung unseres neuen Tarifwerkes aufzuzeigen. Mit TARMED haben wir den Zeittarif eingeführt und damit einer seit langem gestellten öffentlichen Forderung entsprochen. Wir werden für unseren menschlichen und intellektuellen Einsatz entlöhnt und die rein technischen Leistungen sind nur mehr kostendeckend honoriert. Diese Philosophie ist in der Bevölkerung zu hundert Prozent akzeptiert. Mehr noch: wir sind den öffentlichen Erwartungen geradezu vorausgeeilt. Unsere Entlöhnung ist bis auf fünf Minuten transparent und berechenbar. Wir haben damit unsere Hausaufgaben bezüglich Bewertung und Berechnung medizinischer Leistungen mehr als gemacht. Eigentlich sollten wir für diesen neuen Wein auch neue Schläuche fordern: eine Gesundheitspolitik, die sich nicht nur auf das vordergründige Symptom des Kostenfiebers konzentriert, und dann vorschnell zu Antipyretika wie Ausdünnung der Leistungserbringer und Senkung des Taxpunktwertes greift, oder von neuen Therapien redet, die zum Schluss wieder nichts Neues bringen. Auch neue Ärztenetzwerke ändern nichts daran, dass unsere Bevölkerung altert und ständig neue technische Möglichkeiten generiert werden. Der enorme Nachfragedruck bleibt. Diesem wird man sich offen und öffentlich stellen müssen. Er hat mit Kräften zu tun, die jeder in sich selbst vorfindet. Die folgenden Gedanken widerspiegeln deshalb auch eine Selbstreflexion:

Jede Krankheit ermahnt uns, den Tod nicht $\mathrm{zu}$ vergessen. Das Wissen um die Endlichkeit gehört wesentlich zur Qualität unseres Lebens. Eine Medizin, die den Menschen dies vergessen machen will, verfehlt ihr Ziel. Der Arzt versucht, Hilfe $\mathrm{zu}$ geben in einem sich vollziehenden Geschick, welchem der einzelne in seiner individuellen Art entgegengeht. Das macht seine Einzigartigkeit aus. Eine paternalistisch geprägte Medizin kann dieses fragile Gleichgewicht aus Tun und Geschehenlassen empfindlich stören. Im medizinischen Alltag erleben wir immer wieder, dass Patienten und ihre Angehörigen zufriedener sind, wenn sie ihre Krankheit wieder in den eigenen Bedeutungszusammenhang bzw. in die eigenen Hände nehmen dürfen. Der erfahrene Arzt lässt es sehr gerne zu, wenn seine Hilfestellung ihm abgenommen und zur Selbsthilfe umfunktioniert wird. «Patient empowerment» heisst der neue, kraftvolle Begriff, der allerdings etwas kraftlos dem Patienten nur die Hälfte der «power» zutraut, nämlich die Mitbestimmung in Therapiefragen, nicht aber die Mitverantwortung in den Kostenfolgen. Aber um genau diese letzte Mitverantwortung kommen wir nicht herum, wenn wir dem Kostenfieber endlich wirksam begegnen wollen. Ich frage mich, warum dies derart tabuisiert wird! Vielleicht liegt es daran, dass die enormen Erfolge der letzten hundert Jahre die Medizin narzisstisch aufgebläht und den Patienten zur bequemen Erwartung verführt haben, dass eine Welt, die sich zum Mars ausstreckt, gegen seinen persönlichen Dyskomfort doch sicher auch Mittel und Wege zuliesse. Zu gerne lässt sich die 
Ärzteschaft diese Potenz zueignen, und mit der Qualitätsanforderung verinnerlichen wir wie im Doppelpack auch die Kostenverantwortung. Wir werden uns von diesem Anspruch an uns selbst lösen müssen. Unser Teil ist, um den Fassadenbegriff der Qualität einmal wegzulassen, der ehrliche Versuch einer sinnvollen Hilfeleistung, und selbst bei dieser werden wir in unseren Sprechzimmern auch von Nichtwissen und von Scheitern reden müssen. Die Gesellschaft wird, wenn sie den Mut dazu hat, die Rationierungsfrage selber lösen. Auch wird sie nicht um den schmerzhaften Prozess herumkommen, Solidarität und Subsidiarität neu gegeneinander abzuwägen. Nach meiner Meinung wird eine Gesundheitspolitik der Zukunft dem einzelnen Spielraum in der Frage geben, welche medizinischen Leistungen er für sich beanspruchen und einkaufen will. 